\title{
Overview of the Auger@TA project and preliminary results from Phase I
}

Fred Sarazin ${ }^{1}$, Corbin Covault ${ }^{2}$, Toshihiro Fujii ${ }^{3}$, Robert Halliday ${ }^{2}$, Jeffrey Johnsen ${ }^{1}$, Ryan Lorek $^{2}$, Toshiyuki Nonaka ${ }^{3}$, Sean Quinn ${ }^{2,4}$, Hiroyuki Sagawa ${ }^{3}$, Takashi $\mathrm{Sako}^{3}$, Ricardo Sato ${ }^{5}$, David Schmidt ${ }^{6}$, and Ryuji Takeishi ${ }^{7}$ for the Pierre Auger $^{5, *}$ and Telescope Array ${ }^{8, * *}$ collaborations

${ }^{1}$ Dept of Physics, Colorado School of Mines, Golden CO (USA)

${ }^{2}$ Dept of Physics, Case Western Reserve University, Cleveland OH (USA)

${ }^{3}$ Institute for Cosmic-Ray Research, University of Tokyo, Tokyo (Japan)

${ }^{4}$ Dept of Physics \& Astronomy, University of California - Los Angeles, Los Angeles CA (USA)

${ }^{5}$ Pierre Auger Observatory, Malargüe (Argentina)

Full author list: http://www.auger.org/archive/authors_2018_10.html

${ }^{6}$ Karlsruhe Institute of Technology, Karlsruhe (Germany)

${ }^{7}$ Dept of Physics, Sungkyunkwan University, Jang-an-gu, Suwon (Korea)

${ }^{8}$ Telescope Array Project, Salt Lake City UT (USA)

Full author list: http://www.telescopearray.org/research/collaborators

\begin{abstract}
We report on the first results of a unique in-situ experimental cross-calibration effort of the surface detector of the Pierre Auger Observatory and of the Telescope Array experiment (Auger@TA). In the first phase of Auger@TA, we performed surface detector station-to-station comparisons for a collection of extensive air showers landing near the experimental setup and detected by Telescope Array. Beyond the deduced cross-calibration curve between the Water-Cherenkov-based Auger and Scintillator-based TA Surface Detector stations, we also investigate the consistency of their response for individual reconstructed showers. The dataset is currently too small to draw firm conclusions as-of-yet. Hence, phase I data taking will continue even as we gear up for the deployment of an Auger micro-array within Telescope Array as part of Phase II of this work.
\end{abstract}

\section{Introduction}

The Pierre Auger Observatory (Auger) [1] and the Telescope Array (TA) experiment [2] are the two largest UltraHigh-Energy Cosmic-Ray (UHECR) experiments in the world. Both experiments are hybrid detectors, where the Surface Detector (SD) acts as the statistical engine and the Fluorescence Detector (FD) provides, among other things, the energy scale based on calorimetric measurements. After a little over 10 years of data taking, there appears to be a discrepancy of about $10 \%$ between the energy scale of the two experiments [3]. This discrepancy is within the range of the systematic uncertainties of both experiments. However, after re-scaling, a large difference remains at and beyond the flux suppression $[4,5]$. While this could be linked to fundamental differences between the northern and southern UHECR skies, it could also be due to unresolved discrepancies in the way the two experiments are processing Extensive Air Shower (EAS) data. Since 2012, the Auger and TA collaborations have created joint working groups to evaluate the data collected by both experiments. Results from some of those working groups were presented at this conference [6-10].

In 2014, a unique joint experimental effort was initiated to carry out in-situ cross-calibrations between the

\footnotetext{
*e-mail: auger_spokespersons@fnal.gov

**e-mail: placeholder_ta_email@cosmic.utah.edu
}

Auger and TA SDs. The SD of both experiments makes use of different detection media, which are not equally sensitive to the various components of the EAS making it to the ground. The (pre-AugerPrime [11]) Auger SD is based on a Water-Cherenkov Detector (WCD) system, which collects the light produced by charged particles above a certain energy threshold as they slow down in the water volume. The baseline unit for the Auger WCD is an indirect measure of the energy loss in water of a Vertical Equivalent Muon (VEM). The Auger WCD is roughly equally sensitive to the electromagnetic and muonic components of the shower. Separating the two components without additional information is very difficult however. The TA SD is based on plastic scintillators and essentially acts as an indiscriminate counter of particles. The baseline unit for the TA SD is the Minimum Ionizing Particle (MIP) energy loss. The TA SD is predominantly sensitive to the electromagnetic (electrons mostly) part of the shower, because electrons are more numerous than muons (except far away from the core). Given their difference in response, a direct comparison of both SDs is not straightforward and simulations are needed to interpret the correlated signals measured for the co-located Auger and TA SD stations.

The Auger - TA joint experimental effort (Auger@TA) is divided into two phases. The first phase, for which preliminary results are reported here, consists in performing 


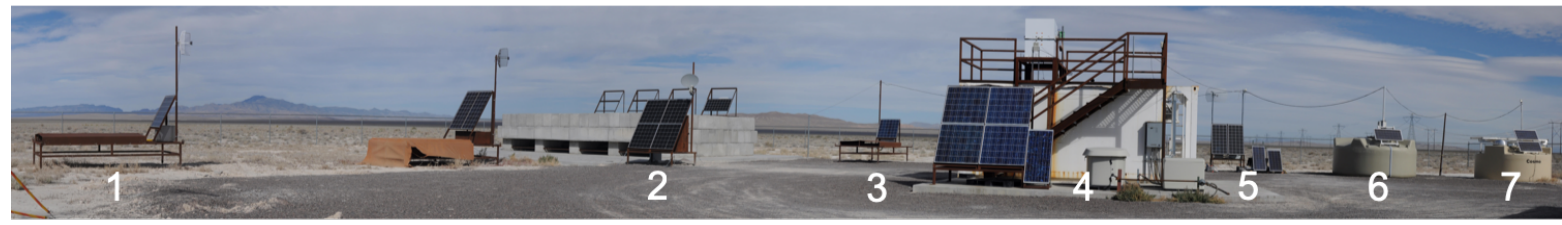

Figure 1. Auger@TA at the TA Central Laser Facility (CLF). 1: DET2421 ("global" TA). 2: LAN comm. and power system. 3: Local TA station. 4: CLF. 5: Auger@TA electronics and solar power system. 6: Auger north (AN) tank. 7: Auger south (AS) tank. The distance between DET2421 and the Auger doublet is about $45 \mathrm{~m}$

station-to-station comparisons with two Auger and two TA SD stations for EASs observed in coincidence. In section 2, we discuss the experimental setup deployed near the TA Central Laser Facility (CLF), a central location within the TA SD. In section 3, we present the basic crosscalibration performed between the Auger (South) and TA SD stations for EASs landing near the CLF that triggered the TA array. In section 4, we carry out a more complex analysis aimed at assessing the consistency of the Auger (South) and TA SD station response for a smaller subset of EASs that are reconstructed by TA. This is followed by conclusions and perspectives. The discussion of the plan for Phase II and the deployment of an Auger microarray within TA are presented in another contribution of this symposium [10]. The Auger@TA joint effort is an official Auger - TA working group.

\section{The Auger@TA Phase I setup}

Figure 1 shows the current setup deployed near the TA CLF. Two Auger stations are deployed as a doublet, separated by about $7 \mathrm{~m}$. One of those stations is the regular Auger (south) SD station deployed at the Pierre Auger Observatory. The other station is a single-PMT prototype originally designed for the Auger North project [12]. The Auger doublet configuration is used to carry out a crosscalibration between the two WCDs in advance of the deployment of the Auger micro-array, which will only consist of Auger North prototypes (see [10]). Data collected with the Auger North WCD prototype will not be discussed further in this contribution. Two TA SD stations are also deployed at the site. The "global" TA station (DET2421) is fully instrumented and operates in normal TA data acquisition mode, although it does not contribute to the global TA trigger decision. The "local" TA station is only partially instrumented and a simple singlethreshold discriminator circuit provides a signal that is used to externally trigger the Auger WCD doublet (for cross-calibration purposes).

Figure 2 summarizes the data flow at the site. The global TA station is connected via wireless communication to the TA data acquisition system. While it does not broadcast local trigger (L1) timestamps, it does listen for readout requests (L2) from the TA data acquisition system and upload its data as requested. The TA trigger is concurrently received via a parabolic antenna installed at the site and connected to a TA electronics kit (e-kit) located in a dedicated enclosure. A firmware modification of the e-kit allows for universal time coordinated (UTC) se- rial messages containing L2 timestamps with microsecond resolution to be communicated to the Auger Single Board Computer (SBC) that handles the Auger side of the trigger readout. The Auger SBC is a Raspberry Pi 2 Model B hardwired to both Auger WCDs (see [13] for details). Software was written to handle the coincidences between the L2 TA triggers and the list of T2 timestamps provided by the Auger WCDs. If a coincidence is found within a $100 \mu$ s time window, the SBC sends a readout request (T3) to the Auger WCDs, and waveforms are collected for further processing. Offline analysis is performed on the data, and true coincidences involving the Auger WCD and global TA stations are separated from accidentals using a narrower coincidence window.

In the most recent implementation of the local trigger, digitized waveforms from the TA local station are collected using a Picoscope 2206B and L1-equivalent signals are generated. Those triggers are combined with the $\mathrm{T} 2$ timestamps from the Auger WCDs to form the local trigger. Following a trigger, L2 (T3) readout requests are sent to the TA (Auger) stations and waveforms are recorded for offline analysis.

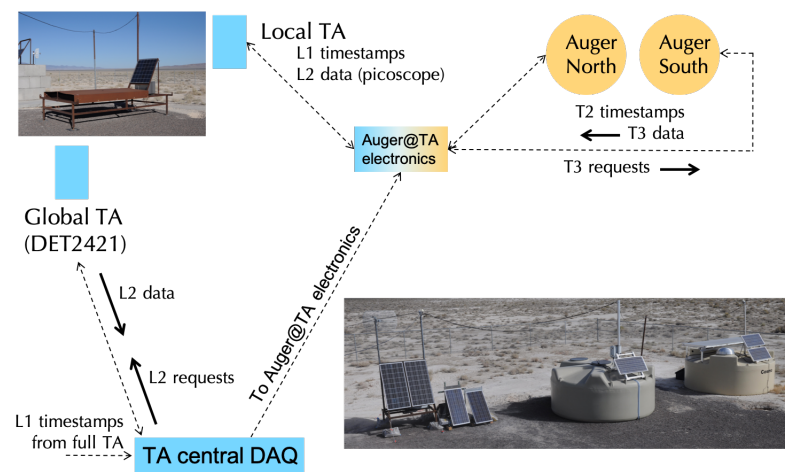

Figure 2. Data acquisition flow of the Auger and TA stations (see text for details).

\section{Auger-TA SD station cross-calibration with EASs detected by TA}

Since operation began, 149 coincidences between the Auger WCD and the global TA stations have been recorded. Three events were discarded due to saturation in either or both detectors. Of the 146 remaining events, only 23 satisfy the TA spectrum quality cuts [14] and have associated reconstruction parameters. Figure 3 shows the correlation between the global TA signal (in MIP) and the 
Auger WCD signal (in VEM) for all 146 events. Shown in black are the 23 events that passed the TA quality cuts.

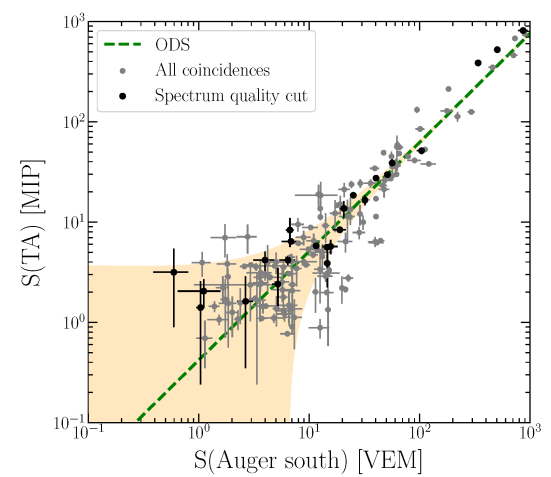

Figure 3. $\mathrm{S}(\mathrm{TA}[\mathrm{MIP}])$ vs $\mathrm{S}($ Auger[VEM]) correlation measured between the TA and Auger stations at the CLF. The black points show the 23 events that passed the TA reconstruction quality cuts. The regression fit presented is performed on all the data.

The signal correlation is not corrected for the distance between the two stations. An orthogonal distance square (ODS) linear regression model is used to fit the full dataset. The result of the fit is consistent with a linear correlation over a large dynamic range (see table 1), and an earlier study showed that the slope of the correlation is consistent with expectation from simulation [13].

Table 1. Regression fit parameters for the ODS log-log data: $\log \left(\mathrm{S}_{T A}[\mathrm{MIP}]\right)=\alpha \log \left(\mathrm{S}_{\text {Auger }}[\mathrm{VEM}]\right)+\beta$

\begin{tabular}{cc}
\hline$\alpha$ & $\beta$ \\
\hline $1.076 \pm 0.037$ & $-0.368 \pm 0.050$
\end{tabular}

\section{Consistency checks}

Beyond the cross-calibration curve discussed in the previous section, a more in-depth station-to-station analysis can be carried out for the 23 coincident events that were reconstructed by TA. The analysis chain is summarized in Figure 4.

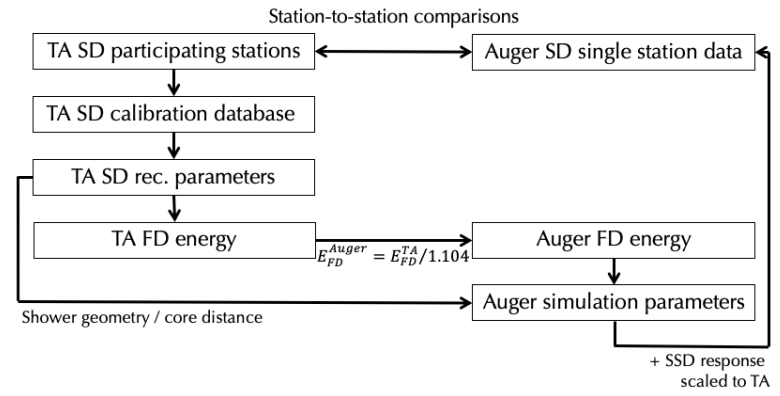

Figure 4. Analysis chain allowing for a test of the consistency of the Auger and TA data collected by Auger@TA phase I (see text for details).
The general idea is to compare the measured (correlated) signals of the Auger and TA SD stations with expectations from EAS simulations. For this work, the analysis was performed using the Auger analysis framework (called "Offline") [15]. The first step consisted in scaling down the TA primary energy by $10.4 \%$ to reflect the difference in energy scale between Auger and TA. Then, using the other TA reconstruction parameters (zenith angle $\theta$, azimuth angle $\phi$ and distance from the core $\rho$ ), a relatively large sample $(N=300)$ of simulated proton showers was created for each event with the CORSIKA [16] air shower simulation tool using two hadronic interaction models (QGSJET-II.04 [17] and EPOS-LHC [18]). Each simulated shower was then reconstructed by Offline. The Auger SD grid was modified to add the two sites corresponding to the positions of the "global" TA and Auger WCD stations near the reconstructed core position. The rest of the SD layout is inconsequential as long as reconstruction is achieved since we only read out the predicted signals at the two locations of interest. Figure 5 shows (top) one of the 23 reconstructed TA event $(E=4.58 \mathrm{EeV}$, $\theta=38.3^{\circ}, \phi=216.7^{\circ}, \rho=820 \mathrm{~m}$ ) and (bottom) one of the simulation realizations of this shower reconstructed by Offline.
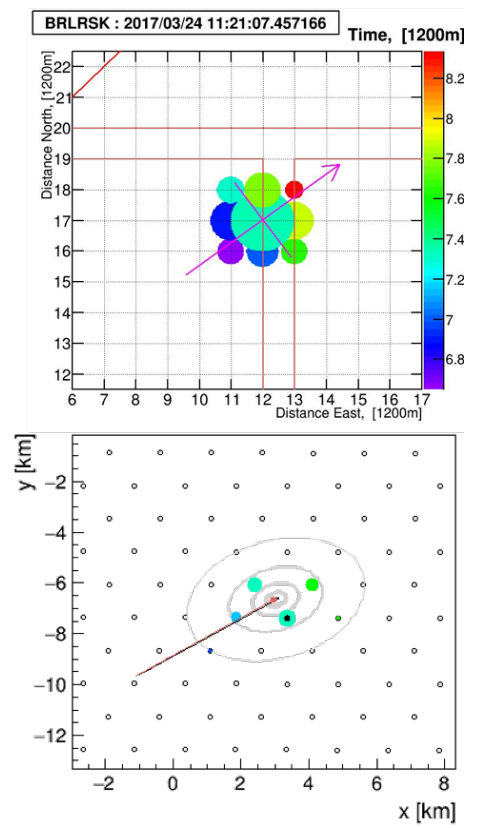

Figure 5. (Top) One of the events reconstructed by TA that landed near the TA CLF. The distances are expressed in unit of separation between TA stations $(1200 \mathrm{~m})$. (Bottom) Simulated Auger shower using the TA reconstruction parameters with the energy scaled down by $10.4 \%$ to account for the difference in energy scale between Auger and TA. The detectors shown on the grid are arranged in the regular Auger triangular pattern $(1500 \mathrm{~m}$ apart).

The most recent development versions of Offline ("trunk") include the upgraded Auger SD, which has a Scintillator Surface Detector (SSD) placed on top of every WCD [11]. Hence, the Offline reconstruction yields a predicted signal for the WCD and the SSD. In this context, we use the SSD as a proxy for the TA SD station, 
and we deduce the predicted TA SD station signal by simply scaling the SSD signal by the ratio of their respective surface areas $\left(3.84 \mathrm{~m}^{2}\right.$ for the SSD, $3 \mathrm{~m}^{2}$ for the TA SD). Moreover, it is now well established that hadronic interaction models underestimate the number of muons in EAS [19]. Following the prescription in [20], the simulation results are corrected for both hadronic models for the muon deficit in simulations. Figure 6 shows the correlation of the measured signals of the Auger and TA SD stations (magenta point) for the previously discussed event, together with the 300 QGSJET-II.04 simulated realizations of the same event before (left) and after (right) correction for the muon deficit in simulations. As can be seen, a better agreement is obtained after correction.

To make systematic comparisons within this dataset, we use the Mahalanobis distance [21] (later referred to as "M-distance") to estimate the distance between the experimental data point and the simulations (assumed to be a representation of the parent distribution the experimental data point belongs to). Figure 7 shows the distribution of M-distances for the $23 \mathrm{TA}$ reconstructed events as a function of three different variables ( $S_{\text {Auger }}, S_{T A}$ and core distance). Additional cuts on the already small dataset are performed. To limit background contributions, we only consider events for which $S_{\text {Auger }}>3 \mathrm{VEM}$ and $S_{T A}>5$ MIP, which correspond roughly to the trigger thresholds for both detectors. Moreover, we only consider TA reconstructed events with $E>3 \mathrm{EeV}$, when the TA trigger efficiency is greater than $80 \%$. This is done to limit the possibility that the experimental signals are upward fluctuations of their respective parent distribution. The discarded data points are shown in grey on Figure 7.
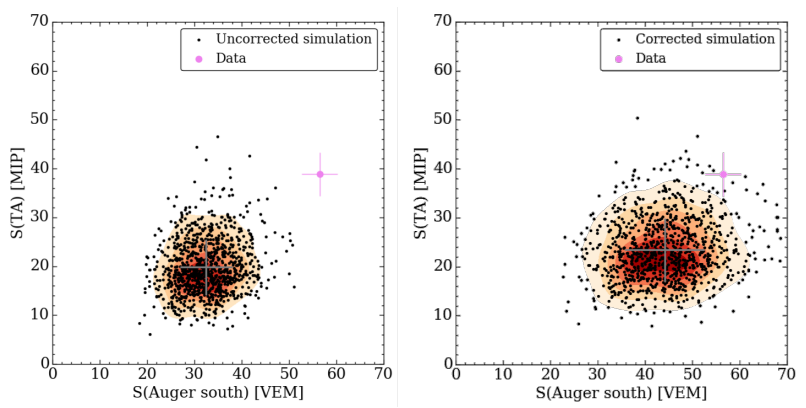

Figure 6. MIP vs VEM signal measured by the two co-located stations for the same event shown in fig. 5 (magenta point) compared with CORSIKA simulations using QGSJET-II.04 before (left) and after (right) correction to account for the muon excess observed in the data.

Overall, shorter M-distances are obtained after accounting for the muon deficit in the simulations, and both hadronic models lead to similar M-distances after correction. Taken at face value, the relatively broad distribution of M-distances appears to indicate that, after energy and muon corrections, discrepancies remain between the measured signals and the predicted signals obtained with the Auger simulation and reconstruction framework using the TA EAS reconstruction parameters. Any conclusion is however mitigated by the low statistics of the current dataset, hence the current analysis of the M-distance dis-
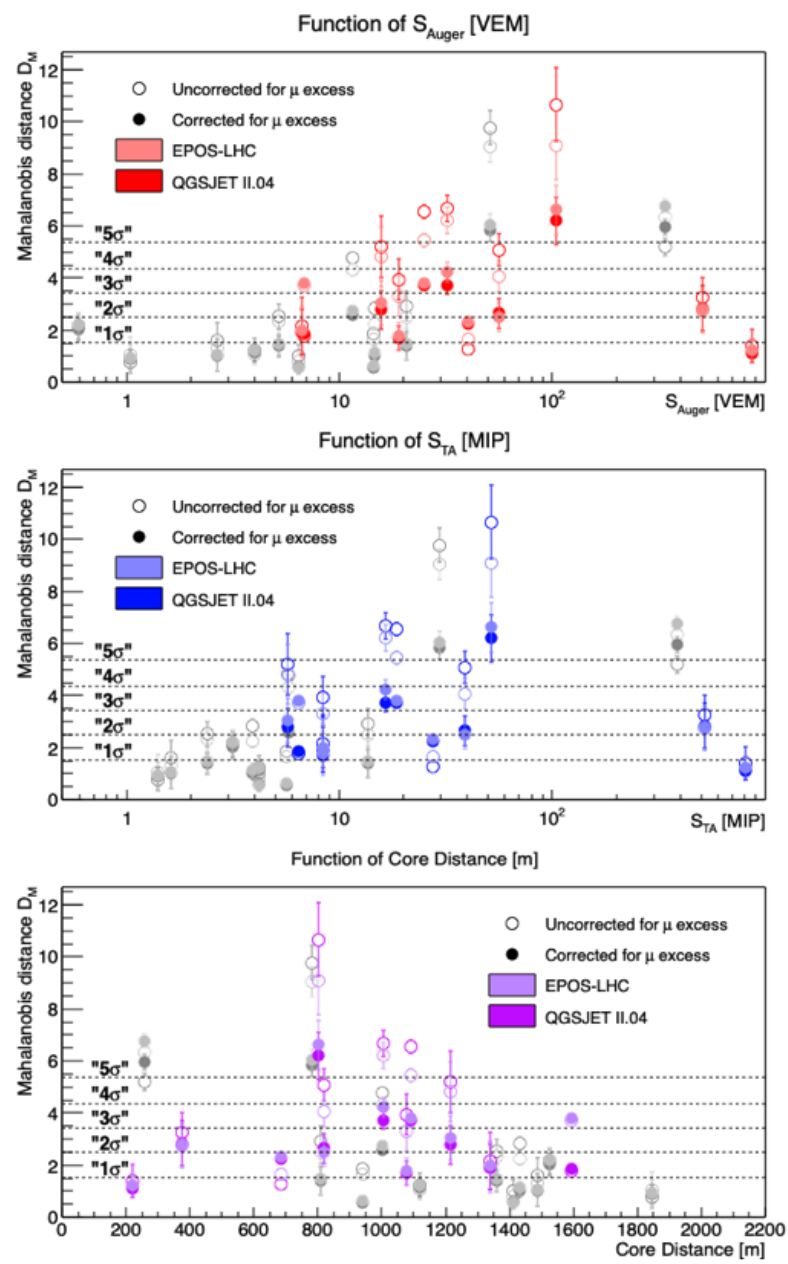

Figure 7. Mahalanobis distance for all the reconstructed events in TA as a function of $\mathrm{S}_{\text {Auger }}$ [VEM] (top), $\mathrm{S}_{T A}$ [MIP] (middle) and core distance (bottom) for two different hadronic models. The grey points show the events that did not pass the additional quality cuts (see text for details).

tribution is ultimately inconclusive. In this context, collection of a more robust dataset will certainly help.

\section{Conclusions \& Perspectives}

In summary, the first phase of Auger@TA is well underway and data taking is still on-going. Beyond the extracted Auger-TA cross-calibration curve, the consistency of the individual station signals is evaluated for a subset of EASs reconstructed by TA. The current statistical limitation of the dataset prevents us from drawing firm conclusions asof-yet. The interpretation is further complicated by the fact that we are performing a "hybrid" analysis using TA reconstructed parameters fed to the Auger simulation and reconstruction framework to evaluate the consistency of the station-to-station response for a selected number of EASs. A similar analysis is being developed using the TA simulation framework in place of the Auger one. It will be interesting to compare the results with what is presented in this contribution. The natural next step however is to deploy an independently-operated Auger micro-array within TA. 
This way, reconstruction parameters for the same EASs could be extracted and analyzed independently by Auger and TA. The result of the parallel analyses could then be compared and possibly cross-calibrated. The plan for this second phase of Auger@TA was presented at this conference [10]. A more in-depth description of Auger@TA Phase I is being prepared for publication [22].

\section{References}

[1] A. Aab et al. [Pierre Auger Collaboration], "The Pierre Auger Cosmic Ray Observatory", NIM A798 172 (2015).

[2] T. Abu-Zayyad et al. [Telescope Array Collaboration], "The surface detector array of the Telescope Array experiment", NIM A 689, 87 (2012).

[3] D. Ivanov for the Pierre Auger and Telescope Array collaborations, "Report of the Telescope Array Pierre Auger Observatory working group on energy spectrum", 35th International Cosmic Ray Conference proceedings, PoS (ICRC2017) 498 (2017).

[4] J. Abraham et al. [Pierre Auger Collaboration], "Observation of the suppression of the flux of cosmic-rays above $4 \times 10^{19} \mathrm{eV}$ ", Phys. Rev. Lett. 101061101 (2008).

[5] R.U. Abbasi et al. [High Resolution Fly's Eye Collaboration],"First Observation of the Greisen-ZatsepinKuzmin Suppression", Phys. Rev. Lett. 100101101 (2008).

[6] D. Ivanov (joint spectrum working group) for the Pierre Auger and Telescope Array collaborations, these proceedings.

[7] A. Yushkov (joint composition working group) for the Pierre Auger and Telescope Array collaborations, these proceedings.

[8] J. Biteau (joint anisotropy working group) for the Pierre Auger and Telescope Array collaborations, these proceedings.

[9] This contribution.

[10] C. Covault (joint Auger@TA working group) for the Pierre Auger and Telescope Array collaborations, these proceedings.
[11] A. Aab et al. [Pierre Auger Collaboration], "The Pierre Auger Observatory Upgrade - Preliminary Design Report", arXiv 1604.03637 (2016).

[12] J. Bluemer for the Pierre Auger Collaboration, "The northern site of the Pierre Auger Observatory", New J. Phys. 12035001 (2010).

[13] S. Quinn for the Pierre Auger and Telescope Array collaborations, "Auger at the Telescope Array: toward a direct cross-calibration of surface-detector stations", 34th International Cosmic Ray Conference proceedings, PoS (ICRC 2017) 395 (2017).

[14] T. Abu-Zayyad et al. [Telescope Array Collaboration], "The cosmic-ray energy spectrum observed with the surface detector of the Telescope Array experiment", Astrophys. J. Lett. 768 L1 (2013).

[15] S. Argiro et al., "The Offline Software Framework of the Pierre Auger Observatory", Nucl. Instrum. Meth. A 5801485 (2007)

[16] D. Heck et al, "CORSIKA: a Monte Carlo code to simulate extensive air showers", FZKA-6019 (1998).

[17] S. Ostapchenko, "Monte Carlo treatment of hadronic interactions in enhanced Pomeron scheme: QGSJET-II model", Phys. Rev. D 83, 014018 (2011).

[18] T. Pierog et al., "EPOS LHC: test of collective hadronization with data measured at the CERN Large Hadron Collider", Phys. Rev. C 92, 034906 (2015).

[19] A. Aab et al. [Pierre Auger Collaboration], "Muons in air showers at the Pierre Auger Observatory: Mean number in highly inclined events", Phys. Rev. D91 032003 (2015).

[20] A. Aab et al. [Pierre Auger Collaboration], "Testing hadronic interactions at ultrahigh energies with air showers measured by the Pierre Auger Observatory", Phys. Rev. Lett. 117192001 (2016).

[21] P.C. Mahalanobis, "On the generalized distance in statistics", Proceedings of the National Institute of Science of India 2 (1936).

[22] Pierre Auger and Telescope Array collaborations, "Auger@TA phase I: in-situ cross calibration of the surface detector stations of the Pierre Auger Observatory and Telescope Array", to be submitted to JINST (2019). 